\title{
Rischi e problematiche medico-legali in epilessia
}

\author{
Chiara Pizzanelli ${ }^{\text {, }}$, Veronica Pelliccia ${ }^{1}$, Renato Galli ${ }^{2}$, Gianni Taccini ${ }^{3}$, Alfonso ludice ${ }^{1}$, Luigi Murri ${ }^{1}$
}

Clinica Neurologica, Dipartimento di Neuroscienze, Università di Pisa

U.O. Neurofisiopatologia, USL 5, P.O. F. Lotti-Pontedera

Associazione Italiana Contro l'Epilessia, Sezione Pisa-Pontedera

\begin{abstract}
For patients with epilepsy coping with the reaction of other people can sometimes be the most difficult part of living with the disease. In many Countries social implications of epilepsy have determined the institution of legislative interventions to patient's rights. Therefore the clinician's responsibility includes helping patients in knowing their rights and the most important laws that have special relevance for people with epilepsy. This article discusses legislative aspects, referring to the Italian law, concerning epilepsy management. Many areas of the life of a person with epilepsy are considered, such as driving, employment, and recreational pursuits.
\end{abstract}

Keywords: epilepsy, legislative aspects, driving, employment

Risks and medical-legal aspects in epilepsy

Pratica Medica \& Aspetti Legali 2010; 4(2): 67-72

\section{INTRODUZIONE}

Fin dall'antichità l'epilessia è stata vista con timore e diffidenza per la presenza di crisi inaspettate, periodiche, in alcuni casi violente e convulsive, accompagnate da stati d'incoscienza. La superstizione e le credenze popolari hanno condizionato la cura dei pazienti con epilessia fino a tempi relativamente recenti, considerando l'epilessia non come malattia, ma piuttosto come una condizione circondata da un senso d'indefinito, di misterioso, di sovrannaturale. Su tali basi si fonda a tutt'oggi lo stigma sociale dell'epilessia, correlato a scarsa informazione e a false credenze. Come affermato dall'epilettologo americano Lennox verso la metà del ventesimo secolo, e tutt'ora attuale, «il soggetto con epilessia soffre per tutto ciò che la malattia comporta a livello sociale più che per la malattia in sé». In effetti, le conseguenze sociali dell'epilessia sono ancora oggi discriminanti e riguardano molteplici aspetti della vita di una persona con epilessia: dipendono dall'età del soggetto, dal suo stile di vita, dal sesso e riguardano le relazioni sociali, la scuola, il lavoro, le attività ricreative e la patente di guida.

La discriminazione in molti casi inizia in età scolare ed è spesso legata allo scarso livello di informazione e di conoscenza da cui derivano l'ansia e la paura di rapportarsi a un bambino con epilessia che ha come conseguenze emarginazione e disagio. Un aspetto da considerare in età adulta è quello relativo all'inserimento lavorativo; l'epilessia costituisce allo stesso tempo una causa di invalidità quando associata a disabilità gravi, controindicazione a svolgere determinate professioni e motivo di inserimento in categorie protette. Un ulteriore aspetto in cui l'epilessia può influenzare la vita del soggetto è rappresentato dalla patente di guida.

Nei vari Paesi del mondo occidentale le implicazioni sociali conseguenti all'epilessia costituiscono la ragione di una serie di interventi anche legislativi a difesa del malato, in particolare a sostegno della persona con disabilità e contro la discriminazione. 
In effetti, in tema di epilessia e legge, molti aspetti della vita meritano attenzione: i compiti di assistenza del medico, la patente di guida, il lavoro e le relazioni sociali. Il presente articolo esamina le implicazioni legali di questi argomenti specifici. Infine, dato che un argomento di interesse in ambito di dibattimenti in tribunali penali è rappresentato dal possibile ruolo delle crisi come momenti di sospensione della coscienza nell'attenuare la colpa, l'ultimo paragrafo costituisce un breve sommario concernente questi aspetti.

\section{COMPITI DI ASSISTENZA}

Compito del neurologo è quello di assistere adeguatamente il soggetto con epilessia aiutandolo in prima istanza a capire la propria malattia: a comprendere per esempio il fatto che le crisi possano essere secondarie a una lesione o meno, che si presentino inaspettate, che in alcuni casi siano precedute da sintomi "premonitori", come l'aura. Il compito successivo è quello di impostare adeguatamente la terapia antiepilettica. L'epilessia è infatti una malattia per la quale nella maggior parte dei casi è possibile ottenere un soddisfacente controllo delle crisi e a questo fine sono disponibili numerosi farmaci. La scelta del farmaco deve basarsi sulla diagnosi sindromica e sulle individuali caratteristiche cliniche, sociali e demografiche del paziente. Larte medica consiste nella scelta di una terapia personalizzata per ogni specifico soggetto (ad es. un bambino, un anziano, una giovane donna, una donna con desiderio di maternità, un paziente che sta assumendo altri farmaci per comorbilità, ecc.).

Come in tutti i campi della medicina, anche per quanto riguarda l'epilessia il medico ha una responsabilità legale verso il paziente denominata "compito di assistenza" in relazione alla quale ci si aspetta che fornisca assistenza ottimale entro i suoi livelli di competenza. Tale assistenza deve rispettare gli standard etici e clinici della medicina necessari per soddisfare le esigenze del paziente. Dell'assistenza al paziente fa parte una componente fondamentale rappresentata dal consenso informato. È assiomatico postulare che la cura del paziente è la concordanza tra quanto il medico propone e quanto il paziente accetta. Laccettare si fonda sul presupposto che il paziente abbia ricevuto l'informazione necessaria su cui basare la decisione dell'accettare. L'inizio di una terapia antiepilettica rappresenta pertanto un patto di fiducia in cui il paziente, informato dal medico dei teorici benefici ed effetti collaterali della terapia, concorda consapevolmente nell'intraprenderla.

A parte i compiti di assistenza, incombenza del neurologo da un punto di vista etico e morale, ma anche legale, è anche quella di informare il soggetto dell'esistenza di normative che riguardano vari aspetti della vita pratica, perché il paziente conosca la possibilità di ottenere agevolazioni (esenzione dal pagamento di ticket sanitari per alcune prestazioni, agevolazioni nel mondo del lavoro, ecc.) e i limiti inerenti la diagnosi di epilessia (limitazioni sulla patente, controindicazioni allo svolgimento di alcuni lavori o di alcuni sport, stile di vita).

\section{LA PATENTE}

Come brevemente accennato nell'introduzione, la possibilità di guidare costituisce per le persone con epilessia un aspetto che influenza in modo rilevante la qualità di vita in termini non solo di autonomia personale, ma anche di possibilità di impiego nel mondo del lavoro.

D'altra parte le crisi epilettiche che si verificano alla guida sono spesso causa di gravi incidenti stradali. Numerosi studi dimostrano che i tassi di incidenti stradali sono solo lievemente maggiori nei soggetti con epilessia rispetto alla popolazione generale e comunque sovrapponibili a quelli di gruppi di soggetti con altre patologie croniche, come il diabete e le malattie cardiache. Complessivamente, il numero di incidenti occorsi a persone con epilessia è esiguo se confrontato con il numero di incidenti dovuti ad altre cause prevedibili, come il consumo di alcol, responsabile, da solo, del 5-10\% degli incidenti stradali.

I vari Paesi europei ed extra-europei hanno prodotto normative che consentono di guidare solo alle persone che presentino soddisfacente controllo delle crisi epilettiche. Tuttavia, non c'è uniformità tra i diversi Paesi su cosa si intenda per "soddisfacente controllo delle crisi”. In Italia secondo la normativa vigente, la concessione della patente - limitata alle categorie A e B - è consentita solo a quei pazienti che non presentino crisi epilettiche da almeno due anni, indipendentemente dalla semiologia critica e dall'effettuazione di terapie antiepilettiche. Tale condizione deve essere verificata dalla commissione medica locale sulla base di certificazione redatta dal neurologo appartenente a strutture pubbliche che segue il paziente. La validità della patente non può essere superiore a due anni, in seguito ai quali occorre il rinnovo con le stesse modalità. Invece la patente di guida delle categorie $\mathrm{C}, \mathrm{D}$ ed $\mathrm{E}$ non può essere rilasciata nè confermata a coloro che sono affetti o che hanno sofferto in passato di epilessia.

Rispetto agli altri Paesi, la normativa italiana appare sicuramente piuttosto rigida e assai limitante per i malati. L'intervallo libero da crisi è infatti quello più lungo richiesto; nella maggior parte degli altri Paesi europei è sufficiente un intervallo di un anno (Belgio, Olanda, Danimarca, Norvegia, Finlandia, Francia, Germania, Regno Unito, Irlan- 
da, Spagna, Svizzera), mentre solo in pochi Paesi, oltre all'Italia, sono necessari due anni (Svezia, Portogallo, Croazia, Slovenia, Estonia). Negli Stati Uniti l'intervallo libero è diverso nei vari stati: per 22 stati è di un anno, per 16 di sei mesi e per 6 stati di soli tre mesi.

Inoltre, la maggior parte degli altri Paesi applica in modo più elastico la normativa stessa, distinguendo i diversi tipi di crisi: ad esempio se le crisi si presentano in sonno o al risveglio, essendo intuibile che la probabilità che si verifichino crisi alla guida in un soggetto con sole crisi morfeiche è ragionevolmente bassa. Infine, mentre in Italia è proibito al soggetto che abbia una storia di epilessia, anche se libero da crisi, il conseguimento della patente di categorie C, D ed E, in alcuni Paesi (Belgio, Francia, Germania, Olanda, Regno Unito, Spagna, Danimarca) sono consentite alcune tipologie di patente a patto che i soggetti siano liberi da crisi da molti anni, non assumano al momento farmaci antiepilettici e presentino tracciati elettroencefalografici ed esami di neuroimmagine nella norma.

In considerazione della severità della normativa attuale, è stata proposta nel gennaio 2007 una modificazione della legge relativa alle normative vigenti riguardanti l'epilessia e la patente. Questa modificazione si inscrive nell'ambito di una proposta di legge molto più ampia che riguarda non solo la patente, ma si estende anche a definire norme per il riconoscimento della guarigione del soggetto con epilessia per il conseguimento di una piena cittadinanza e integrazione sociale. La novità di maggior rilievo è che per la prima volta si prevede il concetto di guarigione e quindi la possibilità di cessazione di tutte le limitazioni derivanti dal precedente stato patologico. Inoltre si riduce dagli attuali due anni a un anno il periodo di libertà da crisi necessario per ottenere/rinnovare la patente. Tuttavia, un punto rilevante che risulta modificato rispetto alla normativa corrente è l'obbligatorietà di comunicare formalmente alle autorità competenti il verificarsi di una crisi da parte del medico che l'ha accertato per i relativi conseguenti provvedimenti; questi ultimi sono, secondo la propo- sta di legge, rappresentati da un lato dalla sospensione della patente e dall'altro dal diritto a una serie di benefici riguardanti l'inserimento lavorativo e la mobilità sul lavoro per le persone che, a causa della loro condizione patologica, non sono abilitate alla guida di un veicolo.

Benché tale proposta sia pregevole nel tentativo di equiparare l'Italia agli altri Paesi europei nella lunghezza del periodo richiesto come libero da crisi per il conseguimento/rinnovo della patente (un anno anziché due), ci sembra in realtà che essa muova aspetti molto delicati dal punto di vista medico-legale. Infatti, il medico che ha in carico il soggetto con epilessia si troverebbe obbligato a eseguire una comunicazione formale di crisi alla motorizzazione. In tal modo si andrebbe incontro a una violazione della privacy, ma soprattutto potrebbe venir meno il rapporto di fiducia tra medico e paziente, con la possibile conseguenza della negazione da parte del paziente stesso di avere crisi nel timore delle conseguenze di tale affermazione.

Nei Paesi dove tale obbligo per i medici è presente, spesso i pazienti cambiano medico fino a che non ne trovano uno che dissenta dalla normativa e che pertanto in un certo senso sia disposto a essere "alleato" del paziente, nonostante questo comporti una violazione delle legge.

\section{IL LAVORO}

Nel 65-70\% dei casi l'epilessia non determina alcuna compromissione della capacità lavorativa e i soggetti sono in grado di fornire prestazioni uguali a quelle del resto della popolazione. Tuttavia, nonostante che la Commissione per l'Occupazione dell'International Bureau for Epilepsy (IBE), l'organizzazione internazionale delle associazioni laiche, raccomandi una politica non discriminante nell'assunzione di persone con epilessia, molti dati sull'occupazione indicano che le persone con epilessia possono presentare problemi sia nel trovare che nel mantenere un'occupazione.

Le cause che possono provocare difficoltà lavorative sono molteplici. Un ruolo determinante è svol-

Forme di epilessia

Percentuali di invaliditò

\begin{tabular}{|lc|}
\hline Epilessia generalizzata con crisi annuali in trattamento & $20 \%$ fisso \\
\hline Epilessia generalizzata con crisi mensili in trattamento & $46 \%$ fisso \\
\hline Epilessia generalizzata con crisi plurisettimanali/quotidiane in trattamento & $100 \%$ fisso \\
\hline Epilessia focale con crisi in trattamento & $10 \%$ fisso \\
\hline Epilessia focale con crisi mensili in trattamento & $41 \%$ fisso \\
\hline Epilessia focale con crisi plurisettimanali/quotidiane in trattamento & da 91 a $100 \%$ fisso
\end{tabular}

Tabella I. Percentuali di invalidità riconosciute al soggetto epilettico in relazione alle diverse forme di epilessia e alla frequenza degli episodi critici 
to da alcuni fattori che possono presentarsi talora associati con l'epilessia: insufficienza mentale, disturbi psichici e comportamentali, deficit motori e sensoriali. Anche la gravità e la frequenza delle crisi, il livello di istruzione, l'isolamento sociale, oltre che il pregiudizio sociale e la scarsa informazione sono tutti fattori da considerare.

In Italia la persona con epilessia è tutelata in ambito lavorativo mediante il riconoscimento dell'epilessia come malattia invalidante e pertanto appartenente alla categoria delle invalidità civili. Invalidi civili sono i cittadini affetti da minorazioni congenite o acquisite, sia di natura fisica sia psichica, che comportano un danno funzionale permanente. La Gazzetta Ufficiale n. 43 del 1992 ha pubblicato una tabella che definisce, in relazione alle diverse forme di epilessia e alla frequenza degli episodi critici, il riconoscimento di diverse percentuali di invalidità (Tabella I).

$\mathrm{Al}$ di là delle percentuali di invalidità, la definizione di invalido civile è connessa con la misura della capacità lavorativa del soggetto. Le persone cui venga accertata una riduzione della capacità lavorativa in misura pari o inferiore a $1 / 3$ (da 0 a $33 \%$ ) non sono riconosciute invalide. Quando invece la riduzione della capacità lavorativa è in misura superiore a $1 / 3$ (da 34 a $66 \%$ ), la persona è riconosciuta invalida. Coloro ai quali sia riconosciuta una riduzione della capacità lavorativa in misura pari o superiore al 46\% in età compresa tra 18 e 65 anni hanno diritto al collocamento obbligatorio, iscrivendosi negli elenchi delle "categorie protette". L'obbligo di assunzione riguarda i datori di lavoro privati, le Amministrazioni, le Aziende e gli Enti pubblici con più di 35 lavoratori. Quando sia stata accertata una riduzione della capacità lavorativa in misura superiore ai $2 / 3$ (dal $67 \%$ al $99 \%$ ), le persone con epilessia hanno diritto al collocamento obbligatorio o, nel caso non siano collocate sul lavoro, a un assegno mensile di assistenza. Le persone con inabilità lavorativa al $100 \%$ hanno diritto a un collocamento obbligatorio o, nel caso non siano collocate, a una pensione di inabilità. Visto che la maggior parte dei soggetti con epilessia non è invalido, la questione dell'epilessia nel mondo del lavoro ha un certo peso. Il datore di lavoro, nel valutare una persona con epilessia con il supporto del medico del lavoro, dovrebbe tenere presente che esistono differenti crisi epilettiche e che la capacità lavorativa del soggetto dovrebbe essere valutata in stretta connessione con le caratteristiche della sindrome epilettica e del tipo di crisi. Inoltre alcune sindromi epilettiche possono essere completamente controllate con una terapia farmacologica adeguata che in molti casi non ha alcun effetto negativo sulla vigilanza e sull'attenzione, non riducendo pertanto le capacità lavorative del soggetto. In questi casi una discriminazione sul lavoro, come talora capita che venga riferito dai pazienti nel corso del consulto medico, appare del tutto ingiustificata.

\section{LE INTERAZIONI SOCIALI}

Viene riportato in letteratura come l'epilessia abbia avuto, soprattutto negli anni passati, ma in alcuni casi ancora oggi, ripercussioni negative in numerosi ambiti della vita sociale e relazionale. Per la persona con epilessia si può parlare di isolamento sociale, intendendo una condizione nella quale il paziente preferisce evitare di relazionarsi con gli altri nel timore costante che si possa verificare una crisi. In questo caso il paziente vive la propria malattia come una "spada di Damocle" e del resto, non essendo in alcun modo prevedibili le crisi, può vivere con ansia la propria condizione, preferendo evitare relazioni con le altre persone. Nelle relazioni sociali giovanili, età in cui si riscontra peraltro un picco di incidenza dell'epilessia, la malattia può rappresentare un ostacolo, complicando l'inserimento all'interno di un gruppo, anche per il rifiuto da parte delle persone di condividere un viaggio, uno sport o un altro momento di svago con una persona con epilessia.

Incredibilmente, per molto tempo, l'epilessia ha costituito una preclusione al matrimonio e ad avere figli; ancora oggi è stato dimostrato che la frequenza dei matrimoni è più bassa per le persone con epilessia con, al contrario, una più alta percentuale di divorzi. Anche le dinamiche all'interno della coppia sono state spesso influenzate dall'epilessia, alterando il rapporto moglie/marito, trasformandolo, per uno dei due coniugi, in un'assoluta dipendenza dall'altro. Anche la possibilità di avere figli per molto tempo è stata contestata alle persone con epilessia, nella convinzione talora che tale patologia si potesse trasmettere alla prole $\mathrm{o}$ più spesso che una madre con epilessia non fosse in grado di occuparsi dei propri figli.

\section{LE ATTIVITÀ RICREATIVE: SPORT E VIAGGI}

Lo sport è una tematica particolarmente delicata da affrontare parlando di epilessia. Considerando l'elevata incidenza dell'epilessia in età infantilegiovanile, è una questione con la quale spesso il soggetto con epilessia si trova a dover fare i conti. In generale, in Italia esistono normative codificate che regolano la certificazione di idoneità sportiva. Tali normative distinguono l'attività sportiva in agonistica (D.M. 18/2/82), nella quale il giudizio di idoneità spetta al medico dello sport e in molti casi si avvale della richiesta di accertamenti, e in non agonistica (D.M. 28/2/83), nella quale il giudizio di idoneità spetta al medico di medicina generale. Nel caso di attività sportiva non agonistica non sono previsti accertamenti specifici, ed è il medico di medicina generale che valuta attraverso un'accurata visita medica l'assenza di controindicazioni all'attività sportiva. 
Nel caso di attività agonistica, gli sport vengono divisi in sport ad alto e a basso impegno cardiovascolare e respiratorio, e gli accertamenti richiesti sono volti a verificare l'integrità dell'apparato cardiovascolare e respiratorio. In alcuni sport tuttavia sono richiesti tra i vari accertamenti da praticare anche l'esecuzione di una visita neurologica e di un EEG per escludere eventuali controindicazioni neurologiche, in particolare per escludere la presenza di epilessia. Si tratta di attività per le quali l'epilessia costituisce una controindicazione assoluta (ad es. sport con veicoli a motore, paracadutismo, pugilato, arti marziali, immersioni subacquee). Al di là di questi sport, non esistono evidenze scientifiche che neghino la possibilità di avere l'idoneità sportiva ai pazienti con epilessia per competizioni sia agonistiche sia non agonistiche. Generalmente l'idoneità è concessa valutando il periodo libero da crisi, il tipo di epilessia, la regolare assunzione della terapia e l'adeguatezza dello sport scelto. Esistono molti sport (ad es. atletica leggera, danza, basket, pallavolo, tennis) nei quali sia a livello agonistico sia non agonistico l'epilessia non costituisce un'assoluta controindicazione. In realtà la complessità del problema è evidente nella pratica clinica nella quale spesso il medico dello sport non ha conoscenze esaustive per contestualizzare adeguatamente l'epilessia del soggetto che richiede l'idoneità e nella quale mancano forse linee guida precise che aiutino il medico a prendere la decisione.

Come lo sport, anche viaggiare rappresenta un aspetto fondamentale nella vita soprattutto delle persone giovani, da un punto di vista di crescita personale e culturale. Tuttavia in alcuni casi anche viaggiare può rappresentare un problema per il soggetto con epilessia: alcune compagnie aeree hanno rifiutato infatti di imbarcare persone che nelle 24 ore precedenti avevano avuto una crisi epilettica, oppure, ed è il caso di un nostro paziente, alcune compagnie aeree hanno richiesto un certificato da parte del neurologo per attestare l'idoneità a volare del soggetto con epilessia. In realtà l'epilessia non costituisce una controindicazione a viaggiare né a viaggiare in aereo, con l'unica accortezza di fare attenzione, in caso di voli transcontinentali, ai cambiamenti di fuso orario per la corretta assunzione dei farmaci antiepilettici.

\section{EPILESSIA E CRIMINOLOGIA}

I comportamenti aggressivi si verificano nella società in molteplici circostanze e le persone con epilessia non sono immuni dal presentarli. Tuttavia, un falso convincimento che correla l'epilessia con i comportamenti aggressivi ha sproporzionatamen- te enfatizzato questa relazione, contribuendo ad aggravare lo stigma sociale già associato a questa malattia; in ambito penale tale distorsione ha determinato in alcuni casi giudiziari anche l'attribuzione di responsabilità di crimini violenti alle crisi epilettiche e ciò appare abbastanza discutibile.

È comunque vero che comportamenti violenti possono essere presenti nel corso di una crisi epilettica e in fase post-critica, essendo in genere caratterizzati da movimenti stereotipati, violenza verso gli oggetti o violenza verbale, aggressività non finalizzata né supportata da azioni o propositi complessi. In linea generale è difficile pensare che in tale condizione di alterazione della coscienza critica o nel corso di una confusione post-critica gli eventuali comportamenti aggressivi di una persona con epilessia possano causare un reato, che richiede invece una notevole componente di progettazione vigile e cosciente. In effetti, gli studi condotti con lo scopo di identificare una possibile correlazione tra epilessia e assenza di responsabilità penale non hanno raggiunto alcun risultato certo. Pochissime sono le sentenze nelle quali un imputato è stato ritenuto non punibile a causa di crisi epilettiche. Diversi sono i comportamenti violenti che talora si osservano in fase intercritica, comuni in persone con disturbi cognitivi e comportamentali secondari a una disfunzione cerebrale di cui l'epilessia può costituire solo un'ulteriore manifestazione.

\section{CONCLUSIONI}

Come in generale nelle varie branche della medicina, anche nell'ambito dell'epilessia il medico ha compiti di assistenza affinché il paziente sia seguito nella fase diagnostica e terapeutica nel miglior modo possibile. Tuttavia, nell'epilessia in particolare, esiste un ambito legislativo che può essere rilevante nel modo di rapportarsi del paziente con la sua malattia e che determina l'influenza della malattia sulla vita sociale del paziente. Dovere del neurologo è pertanto quello di informare adeguatamente il paziente sugli aspetti medico-legali riguardanti l'epilessia, ma anche obbligo etico e morale del neurologo è quello di aiutare il paziente a vivere in un giusto equilibrio la propria patologia considerando l'aspetto giuridico e tutelandosi dai pregiudizi sociali.

\section{DISCLOSURE}

Gli Autori dichiarano di non avere conflitti di interesse di natura finanziaria. 


\section{LETTURE CONSIGLIATE}

Beran RG. Epilepsy and law. Epilepsy Behav 2008; 12: 644-51

- Delany L, Moody JE. Epilepsy, employment and the disability discrimination act. Does legislation make a difference? Seizure 1999; 8: 412-20

- Jacoby A, Austin JK. Social stigma for adults and children with epilepsy. Epilepsia 2007; 48(Suppl 9): 6-9

- Kate Collins TB, Camfield PR, Camfield CS, Lee K. People with epilepsy are often perceived as violent. Epilepsy Behav 2007; 10: 69-76

- Kilinç S, Campbell C. "It shouldn't be something that's evil, it should be talked about": a phenomenological approach to epilepsy and stigma. Seizure 2009; 18: 665-671

- Krumholz A. Driving issues in epilepsy: past, present, and future. Epilepsy Curr 2009; 9: 31-35

- Marsh L, Krauss GL. Aggression and violence in patients with epilepsy. Epilepsy Behav 2000; 1: 160-8

- Parfene C, Stewart TL, King TZ. Epilepsy stigma and stigma by association in the workplace. Epilepsy Behav 2009; 15: 461-6

- Tan CT, Avanzini G. Ethical issues related to epilepsy care in the developing world. Epilepsia 2009; 50: 975-7

- Treiman DM. Epilepsy and violence: medical and legal issues. Epilepsia 1986; 27(Suppl 2): 77-104

\section{CORRESPONDING AUTHOR}

Prof. Alfonso Iudice. Dipartimento di Neuroscienze, Clinica Neurologica. Via Roma, 67 - 56126 Pisa. Tel. 050992562 e-mail: a.iudice@neuro.med.unipi.it 